\title{
Whipple's Disease: A Case Report
}

\author{
Orkide Kutlu $^{\mathrm{a}}$ Selma Şengiz Erhan ${ }^{\mathrm{b}}$ Yasemin Gökden ${ }^{\mathrm{a}}$ Özlem Kandemir ${ }^{\mathrm{a}}$ \\ Tufan Tükek ${ }^{c}$ \\ ${ }^{a}$ Department of Internal Medicine, Okmeydanı Education and Research Hospital, Istanbul, Turkey; ${ }^{\text {b Department of }}$ \\ Pathology, Okmeydanı Education and Research Hospital, Istanbul, Turkey; ' Istanbul University Faculty of Medicine, \\ Istanbul, Turkey
}

\section{Significance of the Study}

- Considering Whipple's disease in the differential diagnosis when faced with a patient with malnutrition can provide early diagnosis and treatment, thus preventing sequelae and death.

\section{Keywords}

Whipple's disease $\cdot$ Tropheryma whipplei $\cdot$ Malabsorption

\section{Abstract}

Objective: Whipple's disease is a very rare systemic infectious disease with an annual incidence of 3 in one million, which may be fatal if not diagnosed and treated appropriately. Clinical Presentation and Intervention: Herein we describe a 49-year-old patient admitted to the hospital with symptoms of severe malabsorption and diagnosed with Whipple's disease. The diagnosis was based on the histopathological findings of small intestine biopsies and PCR analysis. Conclusion: Whipple's disease should be kept in mind while dealing with patients with severe malabsorption, even in the absence of accompanying features of the disease.

C) 2019 The Author(s)

Published by S. Karger AG, Basel

\section{Introduction}

Whipple's disease is a very rare, chronic, systemic infectious disease with an annual incidence of 3 in one million, which may be fatal if not diagnosed and treated appropriately $[1,2]$. Classical Whipple's disease causes severe weight loss with chronic intermittent diarrhea, colic-like abdominal pain, and migratory polyarthritis or arthralgia in large joints. Rarely, there may be clinical manifestations of isolated central nervous system disease, endocarditis, or lung, skin, or eye disorders [3]. The causative agent of Whipple's disease, Tropheryma whipplei, is a gram-positive bacillus $[3,4]$. The mean time between the first symptoms of the patient till the diagnosis of this disease was reported to be 7 years [5]. For that reason, the disease may be life-threatening or may cause some serious sequelae during this period.

Herein we present a patient with the symptoms and signs of severe malabsorption, diagnosed with Whipple's disease within a short time from the onset of symptoms. The disease was severe, and the diagnosis was not delayed; this saved the life of the patient.

\section{KARGER}

E-Mail karger@karger.com www.karger.com/mpp 
Fig. 1. a Foamy macrophages stained for CD68. $\times 400$. b PAS-positive intracytoplasmic eosinophilic granules. $\times 400$. c PASD-positive intracytoplasmic eosinophilic granules. $\times 400$.
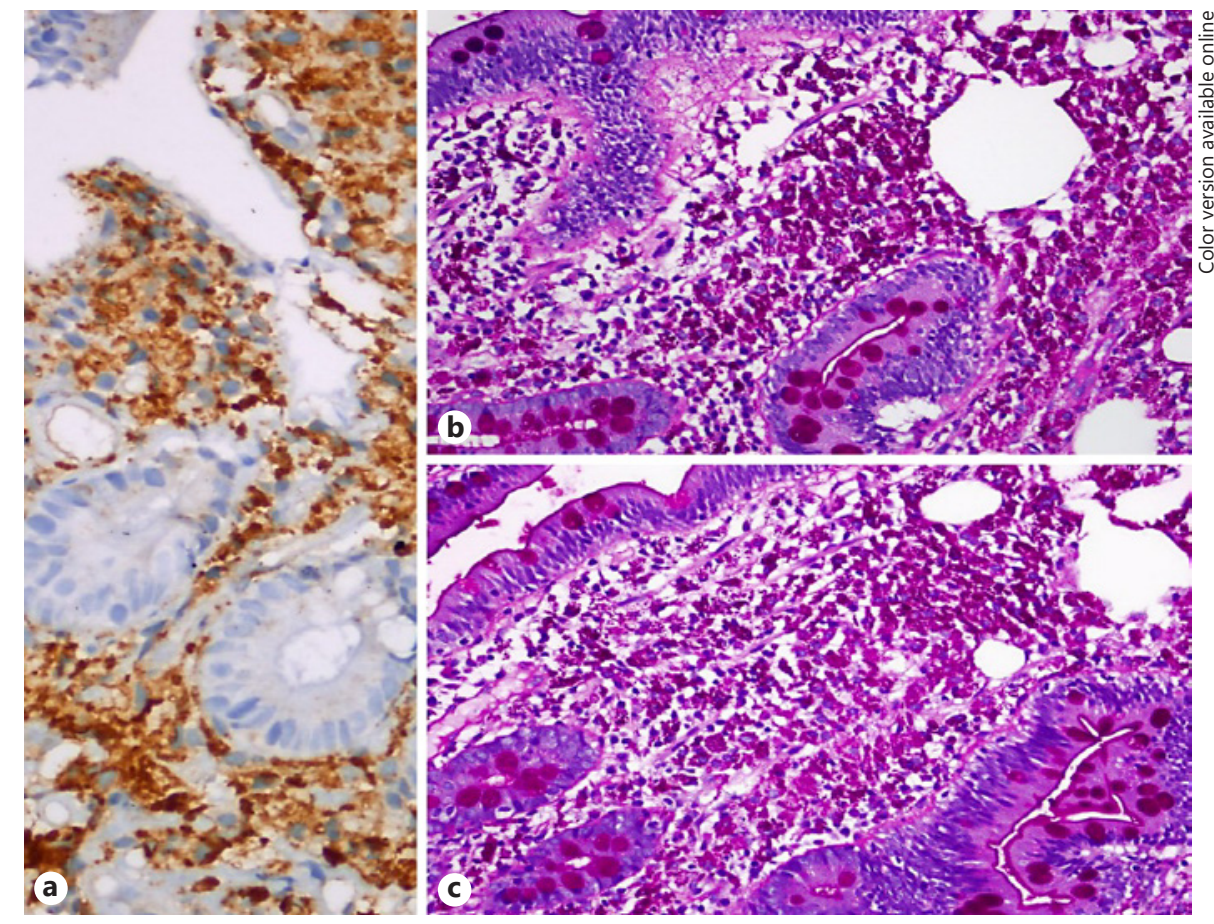

\section{Case Report}

A forty-nine-year-old male garbage collector visited our Internal Medicine outpatient clinic with complaints of weakness, loss of appetite, weight loss, vomiting, diarrhea, and joint pain for about 6 months. His medical history was uneventful. His main symptom was a weight loss of $14 \mathrm{~kg}$ (from 70 to $56 \mathrm{~kg}$, about $20 \%$ of his weight) in the previous 6 months. Physical examination showed that he was conscious and cooperative but cachectic in appearance. His blood pressure was $85 / 60 \mathrm{~mm} \mathrm{Hg}$, with a normal pulse rate and body temperature. There was widespread tenderness on abdominal examination without defense or rebound; his other systemic examination findings were normal. The laboratory data was as follows; hemoglobin: $7.7 \mathrm{~g} / \mathrm{dL}$ (11.5-15.5); white blood cell: $4.1 \times 10^{3} / \mu \mathrm{L}(4.5-10.5)$; C-reactive protein: $67 \mathrm{mg} / \mathrm{L}(0-5)$; erythrocyte sedimentation rate: $50 \mathrm{~mm} / \mathrm{h}$; total protein: $4.8 \mathrm{~g} / \mathrm{dL}$ (6.6-8.7); albumin: $2.8 \mathrm{~g} / \mathrm{dL}$ (3.5-5.2); iron: $15 \mu \mathrm{g} / \mathrm{dL}(67-175)$; calcium: $7 \mathrm{mg} / \mathrm{dL}$ (8.4-10.29); phosphorus: $2.48 \mathrm{mg} / \mathrm{dL}$ (2.7-4.5); and magnesium: $1.78 \mathrm{mg} / \mathrm{dL}(1.8-2.6)$. All other laboratory investigations were within normal limits, including renal and liver function tests and thyroid hormones. Serological markers for HIV and celiac disease and tuberculosis polymerase chain reaction (PCR) were negative. Abdominal ultrasound and computed tomography of the thorax were unremarkable. Abdominal computed tomography revealed a dirty appearance in the mesenteric fatty tissue. During hospitalization, his hemoglobin level gradually decreased to 5.8 g/dL (hematocrit: $19.5 \%$ ), and supportive treatment was initiated with 2 units of erythrocyte suspension and total parenteral nutrition. To determine the etiology of iron-deficiency anemia and malnutrition, upper-gastrointestinal-system endoscopy was done, which revealed a hyperemic, eroded, and yellowish duodenum mucosa: Colonoscopy revealed an edematous appearance in the terminal ileum. Histopathological examinations of the duodenum and terminal ileum biopsies showed a diffuse foamy macrophage infiltration of the lamina propria under the intestinal epithelium, which positively stained for CD68. In the cytoplasm of macrophages, eosinophilic granules that stained positively with periodic acid-Schiff (PAS) and PAS-D were noted (Fig. 1). Fat vacuoles were also observed between macrophages in the lamina propria. In the PCR analysis performed, 16S rRNA sequence analysis confirmed that the causative microorganism was T. whipplei.

With the diagnosis of Whipple's disease, $2 \mathrm{mg}$ /day IV ceftriaxone therapy for 2 weeks was started, followed by twice daily trimethoprim- sulfamethoxazole (160 mg TMP-800 SMX). Clinical improvement was observed within days, and all laboratory findings improved and normalized within months. Histopathological examination of the small bowel biopsy obtained after 6 months of treatment showed a regression in macrophage infiltration of the intestinal subepithelial region and a decrease in eosinophilic granules in macrophages.

\section{Discussion}

In this report, we have presented a middle-aged patient who was diagnosed with Whipple's disease during investigations to determine the etiology of severe malabsorption, which presented with chronic diarrhea, iron-deficiency anemia, and electrolyte disturbances. It is very important to suspect and diagnose this rare, treatable disease as treatment options have to be based on proper diagnosis. 
Classical Whipple's disease is most commonly seen in middle-aged males and in people who work closely with soil; it manifests with prodromal findings of fever, fatigue, and joint disorders [4]. Classical Whipple's disease is characterized by a tetrad of symptoms: arthralgia, weight loss, diarrhea, and abdominal pain. In classical Whipple's disease, prodromal symptoms are generally followed by abdominal pain, nausea, chronic/intermittent diarrhea, and severe weight loss due to malabsorption. Gastrointestinal findings are the most common clinical manifestations in about $90-95 \%$ of the patients [5]. Chronic intermittent polyarthritis is seen in $65-70 \%$ of the patients, which is usually migratory and nonerosive, affecting large joints. The gender, age, and job characteristics of our patient were consistent with the literature. He had prodromal symptoms such as weakness, loss of appetite, headache, and marked discomfort in the joints, which were not specific for Whipple's disease. In our case, the main symptom was chronic diarrhea due to malabsorption, which resulted in severe weight loss; these symptoms improved within months after initiation of the treatment.

In Whipple's disease, involvements of some other organs may be seen. Central nervous system involvement may cause ataxia, seizures, psychiatric disturbances, and dementia. There are also some symptoms defined as pathognomonic for Whipple's disease, for instance oculomasticatory or oculo-facial-skeletal myorhythmia [6]. On the other hand, cutaneous involvement with rashes or papules, culture-negative endocarditis, uveitis/retinitis/ optic neuritis, pulmonary involvement, lymph node involvement, or spondylodiscitis have also been reported in Whipple's disease [7].

A definite diagnosis of classical Whipple's disease is generally made by observing massive infiltration of foamy macrophages containing dense PAS-positive granules in the lamina propria on histopathological examination of the biopsy material obtained from the small intestine. Other characteristic histopathological findings for the disease are small fat collections of the lamina propria and surrounding dilated villi, and ectasia on lymph vessels. In the absence of typical PAS-positive granules, specific antibodies to T. whipplei may be determined in peripheral blood samples, or immunohistochemical studies can be performed for the identification of bacterial $16 \mathrm{~S}$ ribosomal RNA molecules. Rarely, some other tissue biopsies may be required, such as heart valves or synovial fluid, if diagnosis cannot be performed by small bowel biopsy [8]. Endoscopic evaluation of our patient revealed a hyperemic and yellowish appearance in the duodenum mucosa and an edematous appearance in the terminal ileum, suggest- ing infectious or inflammatory small bowel pathology. With the suspicion of Whipple's disease on the pathological evaluation of biopsy materials, PCR analysis revealed the causative microorganism and confirmed the diagnosis of Whipple's disease.

Other infectious causes, inflammatory bowel diseases, connective tissue diseases, hyperthyroidism, and celiac disease should be considered in the differential diagnosis of Whipple's disease. Moreover, other rare causes of PASpositive macrophage infiltration in the small intestine should also be assessed with additional tests for histoplasmosis, Rhodococcus, and HIV-infected mycobacterial disease [9]. In our patient, duodenum biopsy was also obtained for the differential diagnoses of other diseases involving small bowel, and laboratory data were obtained for the identification of other systemic diseases.

In classical Whipple's disease, after treatment with penicillin ( 2 MU IV every $4 \mathrm{~h}$ ) or ceftriaxone (2 g IV single dose daily) for 2-4 weeks, the treatment should be continued with trimethoprim- sulfamethoxazole $(160 \mathrm{mg}$ TMP-800 SMX twice daily) for at least 12 months. Response is monitored by clinical examination. Tests for $T$. whipplei after treatment may be considered if there are new symptoms. Relapses are due to inadequate eradication of microorganisms with initial treatment, and for that reason, higher doses and longer treatment durations are recommended in relapses [10].

\section{Conclusion}

It is still a challenge to diagnose Whipple's disease. Sometimes years elapse between initial findings and characteristic clinical manifestations and diagnosis of the disease. Whipple's disease should be kept in mind in patients with abdominal pain, diarrhea, weight loss, and joint findings. Other rare manifestations of Whipple's disease include immunosuppressive treatment-resistant seronegative arthritis, unknown fever, chronic serositis, myoclonus, ophthalmoplegia, and early onset of cognitive disorders. Clinicians should be aware of this infectious disease in these conditions, as delay in diagnosis may be life-threatening.

\section{Acknowledgement}

We thank Prof. Dr. Barış Otlu for determining the causative microorganism by carrying out the PCR study. 


\section{Statement of Ethics}

Ethics committee approval was not required because our study is a case report.

\section{References}

1 Biagi F, Balduzzi D, Delvino P, Schiepatti A, Klersy C, Corazza GR. Prevalence of Whipple's disease in north-western Italy. Eur J Clin Microbiol Infect Dis. 2015 Jul;34(7):1347-8.

2 Ojeda E, Cosme A, Lapaza J, Torrado J, Arruabarrena I, Alzate L. Whipple's disease in Spain: a clinical review of 91 patients diagnosed between 1947 and 2001. Rev Esp Enferm Dig. 2010 Feb;102(2):108-23.

3 Marth T. Whipple's disease. Acta Clin Belg. 2016 Dec;71(6):373-8.

4 Obst W, von Arnim U, Malfertheiner P. Whipple's Disease. Viszeralmedizin. 2014 Jun;30(3):167-72.

\section{Disclosure Statement}

Dr. Kutlu has nothing to disclose.
5 Marth T. Systematic review: whipple's disease (Tropheryma whipplei infection) and its unmasking by tumour necrosis factor inhibitors. Aliment Pharmacol Ther. 2015 Apr;41(8): 709-24.

6 Anderson M. Neurology of Whipple's disease. J Neurol Neurosurg Psychiatry. 2000 Jan;68(1):2-5.

7 Al-Hamoudi W, Habbab F, Nudo C, Nahal A, Flegel K. Eosinophilic vasculitis: a rare presentation of Whipple's disease. Can J Gastroenterol. 2007 Mar;21(3):189-91.
8 Dolmans RA, Boel CH, Lacle MM, Kusters JG. Clinical manifestations, treatment, and diagnosis of Tropheryma whipplei infections. Clin Microbiol Rev. 2017 Apr;30(2):529-55.

9 Durand DV, Lecomte C, Cathébras P, Rousset $\mathrm{H}$, Godeau P. Whipple disease. Clinical review of 52 cases. The SNFMI Research Group on Whipple Disease. Société Nationale Française de Médecine Interne [Baltimore]. Medicine (Baltimore). 1997 May;76(3):170-84

10 Lagier JC, Fenollar F, Lepidi H, Giorgi R, Million M, Raoult D. Treatment of classic Whipple's disease: from in vitro results to clinical outcome. J Antimicrob Chemother. 2014 Jan; 69(1):219-27. 